The University of San Francisco

USF Scholarship: a digital repository @ Gleeson Library |

Geschke Center

2010

\title{
AutOMathic Blocks: Supporting Learning Games for Young Blind Students
}

Arthur Karshmer

University of San Francisco, akarshmer@usfca.edu

Follow this and additional works at: http://repository.usfca.edu/at

Part of the Disability and Equity in Education Commons

\section{Recommended Citation}

Karshmer, Arthur, "AutOMathic Blocks: Supporting Learning Games for Young Blind Students" (2010). Business Analytics and Information Systems. Paper 19.

http://repository.usfca.edu/at/19 


\title{
AutOMathic Blocks: Supporting Learning Games for Young Blind Students
}

\author{
Arthur Karshmer ${ }^{1}$ and Ken Paap ${ }^{2}$ \\ ${ }^{1}$ University of San Francisco \\ San Francisco, CA USA \\ akarshmer@usfca.edu \\ ${ }^{2}$ San Francisco State University \\ San Francisco, CA USA \\ kenp@sfsu.edu
}

\begin{abstract}
The AutOMathic Blocks system has been developed to help young blind students in the acquisition of elementary math skills. Through the use of Braille labeled blocks, a plastic grid, a touchpad device and any computer, the system has been defined to aid the young student in this most important domain of education. Without these basic skills, students have a much higher probability of not being able to enter math related professions.
\end{abstract}

Keywords: Mathematics, Learning, Blind

\section{Introduction}

In general, the acquisition of math skills is dramatically more difficult than for sighted students. The fact that even introductory arithmetic is a two dimensional representation, complicates the learning ability of young blind students. More advanced math is often what is called a $2 \frac{1}{2}$ dimensional representation - even more difficult for the blind. Even Braille representations of math present the subject in a linear fashion.

The AutOMathic Blocks [1] system obviates these problems by presenting early math in its true form of non-linearity. Through the use of blocks labeled with simple Braille, the learner can tactically examine math problems with their fingers and thereby receive a two dimensional view of their work. It has been demonstrated that tactile input can do the equivalent of mapping tactile information to the visual cortex.

Learning does not end with formal representations of information. Over the recent past, games have been implemented to aid in the learning process. To that end, we have begun to implement simple game playing into the AutOMathic Blocks system. The first game implemented is the traditional tic-tac-toe game for two players. It will be used to help blind students learn how to work in an environment that resembles the environment used by their sighted colleagues. As a further benefit, it will enable 
cognitive scientists to learn more concerning object representation in the mind of the congenitally blind. The remainder of this paper describes several experiments using the games built into the AutOMathic Blocks system to evaluate the usefulness and efficacy of the presentation of math in a two-dimensional form to help visually impaired children learn the critical basics that will prepare them for more complex math later in the educational process.

\section{Testing the conceptual model using}

\subsection{Why is it particularly difficult to learn mathematics without vision?}

Although visually impaired children are developmentally delayed in a variety of knowledge domains the delay is greater for those skills that require the acquisition and maintenance of a two- or three-dimensional problem space in working memory (e.g., the visual-spatial sketchpad component, [2]). This follows because vision directly provides a spatial representation where different identifiable objects can be simultaneously and precisely located in specific locations. Visually perceived space is also very flexible with respect to the way in which objects are attended to and scanned. In contrast, audition (and especially speech) relies heavily on sequential processing. Because even basic mathematics is multidimensional it is very difficult to understand and solve problems with input-output mediums that are inherently linear. Unfortunately computer-based assistive technologies have relied on translating sequences of visual objects (i.e., numbers, letters, symbols, words) to speech. The conceptual model underlying the design of our basic studies and of our prototypes assumes that touch or some combination of touch and audition is the best substitute for providing a multidimensional spatial representation in the absence of vision.

\subsection{Spatial Representations in Vision and Touch.}

Studies aimed at new enabling technology for those with severe visual impairment is always constrained by the number of available participants, especially those who are fluent in Braille. Some of the empirical work we propose uses blindfolded participants with, otherwise, normal vision. This decision goes beyond expediency, as we are interested in basic questions regarding the ability of touch and vision to support mental representations of two-dimensional space. This requires an understanding of the relationship between touch and vision and how that relationship develops. As argued below, this knowledge has implications for differences between congenitally blind populations versus those advantageously blind and why their optimal assistive technology may differ. This section briefly summarizes what is known about the relationship between touch and vision.

A mistaken reason to believe that touch is the best substitute for vision is the common belief that visual perception is learned by concomitant experience with touch 
during infancy. Influential proponents of this view start with Berkeley [3] and Dewey [4] and remain accepted truths by many optometrists and ophthalmologists. However, classic experiments by Rock [5] and his colleagues have shown that when vision and touch are placed in conflict vision captures and dominates touch. With appropriate optical devices inducing a conflict between vision and touch, these experiments show that true size can be underestimated by half, that straight rods can feel curved, and that a square can feel like a narrow rectangle.

A better basis for the expectation that touch leads to mental representations of space similar to those constructed through vision is that some spatial illusions occur in both modalities especially when the experience is produced by active haptic sensing rather than passive tactile sensing (viz., pressing a raised figure onto the skin). Not only does the Muller-Lyer illusion occur when participants freely move their fingers and can simultaneously touch the figures in several places at the same time Casla [6], Millar [7] and Wong [8], but the strength of the illusion varies in the same way as a function of the angle of the arrowhead with respect to the shaft Over [9]. These experiments with sighted, but blindfolded individuals must be interpreted carefully in light of the work described in the previous paragraph showing the vision dominates touch. That is, it may be the case that sighted individuals construct spatial representation from touch by "visualizing" in ways that congenitally blind individuals could not. It has been reported Bean [10] and Hatwell [11] that the congenitally blind also experience the Muller-Lyer illusion, but it would be informative to compare blindfolded sighted and/or advantageously blind individuals to congenitally blind participants on tasks that require the construction of spatial representations that are more similar to those of interest to this project. Another line of studies that supports the similarity of spatial relationships constructed from vision and touch is inspired by Kosslyn's [12] seminal work on visual imaging. All the major experimental effects that supposedly reveal the spatial and non-verbal properties of visual imagery (such as mental rotation, scanning, size/inspection time effects, and selective interference), have now been demonstrated in congenitally blind participants Marmor [13], Carpenter [14], Kerr, [15], Hampson [16], Vecchi [17, Barolo [18] and Aleman [19].

Roberta Klatzky and her associates (e.g., Klatzky [20] have developed a sophisticated model of how both egocentric and allocentric representations of manipulatory space (within arm's reach) can be generated from kinesthetic inputs. An index finger can be returned to a previously sensed target location with impressive precision. A more complex representation that computes second-order parameters allows participants to reproduce (at a new location) either the straight-line distance between two previously and simultaneously sensed finger locations (e.g., left and right index fingers) or the angle of that straight line with respect to the participant's frontal plane with somewhat less precision. Most of the work on manipulatory space involves passive tactile or kinesthetic sensing rather than the active movements that occur when users manipulate the AutOMathic Blocks. Spatial representations constructed from active haptic sensing and prehension should be more precise. Another important difference between this body of research and our concerns is that previous studies on manipulatory space is surprisingly bereft of objects to manipulate 
and integrate into the spatial map. This is not true of the large body of work on locomotor space that is concerned with way finding and cognitive maps.

One series of experiments Gaunet [21] and Thinus 22] have investigated the spatial relations of haptically identified objects in manipulatory space. For example, objects with cross-sections corresponding to squares, circle, and triangles were spontaneously explored in a first phase. Following a spatial change participants reexplored the layout and had to verbally describe the modification. Although early blind participants made more errors than both late blind and blindfolded sighted groups the difference in performance appears to be caused by differences in exploratory patterns. Early blind participants relied more on visiting a sequence of multiple objects whereas the other groups relied more on moving back and forth between the same two objects several times before moving to another object. The authors speculate that the coexistence of these two components (large exploratory cycles and back-and-forth movements) may be interpreted as corresponding to a strategy that consists of roughly comprehending the holistic configuration and subsequently refining this knowledge through more detailed analysis. Because early blind participants used a different mix of strategies the differences in performance may result more from differences in exploratory patterns than any immutable limitation on their mental representations of objects in two-dimensional space.

\section{Further Support for the Conceptual Model}

In summary of the previous section, the research on active haptic sensing is consistent with the hypothesis that blind or blindfolded participants can construct a good mental representation of the layout of objects in two-dimensional space, but the ideal basic research remains to be done. The ideal would map onto the environment of the AutOMathic Blocks system and consequently include these features:

a. Employ manipulatory space rather than locomotor space

b. Identification of objects (attributes, shapes, or characters) that fit on a fingertip

c. Spatial relationship between objects is task relevant

d. Spatial representations are maintained in working memory, but can be refreshed or updated through haptic exploration

These critical features are present in blindfolded versions of the familiar tic-tac-toe game that we propose to use as an experimental paradigm and is operational on the AutOMathic Blocks system. The basic question is the relative ability to form a mental representation of two-dimensional space either based on touch (Touch Alone condition) or a combination of touch and voice (Touch+Voice condition) when visual information is not available. A third condition (Abstract Coordinate) uses speech to specify coordinates and requires a more abstract (amodal) spatial representation that is not directly supported by sensory input from physical locations. 
In the Touch Alone condition the participant places one of his/her raised " $\mathrm{X}$ " tiles in one location of a $3 \times 3$ grid that is similar to the larger workspace grid of the AutOMathic Blocks prototype. The "opponent' (experimenter) responds by placing a raised "O" tile in an open location and then passively moving the participant's finger to that location. To keep the game interesting and the participant motivated, the opponent's responses are based on a script that controls for the probability of the participant winning. The participant is free to review the position of the tiles on the board at any time when it is his or her turn. Dependent measures include average decision time (turn duration), the number of times the identity or location of previously played pieces are reviewed, and outcome (proportion of wins, ties, and losses). The Touch Alone condition is, of course, a scaled down version of the AutOMathic Blocks Touch Alone prototype. Having only two characters (X's and O's) enables one to bypass the complications of Braille fluency, to use readily available sighted participants, and to use a less complex task (compared to math problems) that, nonetheless, places a premium on maintaining a two-dimensional spatial representation of the location of several objects.

In the Touch+Voice condition the raised tiles with X's or O's are not used. Rather, each location on the $3 \times 3$ grid contains a soft response key. When the participant presses a key in a specific location $s /$ he says " $X$ " and a voice recognition system records an $\mathrm{X}$ at the pressed location. On the opponents turn the participant's finger is passively guided to the response location and the opponent says "O" recording the identity of the character at that location. On his or her turn the participant can review the contents at any location by pressing the key and hearing "X", "O", or "blank". Note that this condition involves the same movements and physical locations on the $3 \times 3$ grid as the Touch Alone condition and, consequently, provides direct information about the contents of real locations in space. The only difference is whether the identity of the contents at those locations is determined by touch (e.g., feeling a raised X) or by voice (e.g., hearing a spoken X).

The third condition (referred to as Abstract Coordinate) in this experiment requires participants to construct and maintain two-dimensional spatial representations of the tic-tac-toe board without input from any physical objects or locations. In that sense it is more abstract and more amodal than the first two conditions. The board and pieces are referenced by letter (Columns A, B, and C) and number (Rows 123 ) coordinates. Thus, the participant could designate the placement of one of his $\mathrm{X}$ tokens in the upper-left location by saying "A 1". Likewise, the opponent responds with the coordinate of an open location (e.g., "A 3"). As usual, on his or her turn the participant can review the contents at any coordinate: "What's in B 2?"

In order to control for individual differences a repeated measures design will be used with 30 subjects participating in each of the three conditions: Touch Alone, Touch+Voice, and Abstract Coordinate. The participants will be volunteers drawn from the SFSU Psychology Department participant pool. Each of the three sessions will last about an hour and take place on a different day to minimize carryover effects. The order of the three conditions will be counterbalanced across participants. 
A repeated-measures ANOVA with appropriate a priori comparisons will be used to test the following hypotheses. We expect the touch component of the Touch Alone and Touch+Voice conditions to be highly useful in constructing and maintaining the two-dimensional representation of the workspace. If these conditions are not substantially superior to the Abstract Coordinate condition then our underlying conceptual model for the design of our prototypes is undermined. Another important comparison is between the Touch Alone and Touch+Voice conditions as the Touch Alone condition is analogous to our original Braille-based AutOMathic Blocks prototype whereas the Touch+Voice condition is analogous to a second prototype described later that substitutes voice identification for Braille. If the Touch+Voice condition is comparable to the Touch Alone condition, there would be basic research support for the viability of a touch plus voice prototype that can be used by the many visually impaired individuals who are not fluent Braille readers. The literature reviewed above clearly supports our design hypothesis regarding the utility of manipulatives and haptic sensing of spatial location, but as described at the very onset of this section there is a gap between the many decades of basic research on the various sensory modalities and their application to the design of a system such as AutOMathic Blocks. The experiment described in this section should fill that gap.

\section{Summary}

Through the use of simple games, we expect to get a clearer understanding of the mapping from tactile input to a special representation of mathematics in the visual cortex. As noted, earlier experiments in this domain indicated a strong correlation between tactile input and visual representation (see Muller-Lyer Illusion). In this experiment, a tactile copy of the visual illusion was presented to blindfolded subjects by pressing the solid object on their skin. The results clearly showed that the tactile representation elicited the same responses as the visual representation.

In the current case, we are interested to determine if the same result can be demonstrated in the tactile presentation of math as achieved in the visual presentation. This finding could lead to a whole new set of teaching aids for the visually impaired.

\section{References}

1. Karshmer, A.I., AutOMathic Blocks - An Automated Systems to Teach Math to K-12 Children with Severe Visual Impairment, $1^{\text {st }}$ Annual Learning with Disabilities Conference, Dayton, OH, July, 2007

2. Baddeley, A. D., \& Hitch, G. J. (2007). Working memory: Past, present....and future? In N.Osaka, R. Logie \& M. D'Esposito (Eds), Working Memory - Behavioural \& Neural Correlates. Oxford University Press.

3. Berkeley, G. (1709/1975) Berkeley, George. Philosophical Works, Including the Works on Vision. Edited by Michael R. Ayers. Everyman edition. London: J. M. Dent.

4. Dewey, J. (1899). Psychology, Harper \& Brothers. 
5. Rock, I. (1975). An Introduction to Perception. New York: Macmillan Publishing Co.

6. Casla, M., Blanco, F., \& Travieso, D., (1999). Haptic perception of geometric illusions by persons who are totally congentitally blind. Journal of Visual Impairment and Blindness, 93, 583-588.

7. Millar, S. \& Al-Attar, Z. (2002). The Muller-Lyer illusion in touch and vision: Implications for multi-sensory processes. Perception \& Psychophysics, 64, 353-365.

8. Wong, T. S. (1975). The respective role of limb and eye movements in the haptic and visual Muller-Lyr illusion. Quarterly Journal of Experimental Psychology, 27, 659-666.

9. Over, R. (1966). A comparison of haptic and visual judgments of some illusions. American Journal of Psychology, 79, 590-595.

10.Bean, C. H., (1938). The blind have "optical illusions". Journal of Experimental Psychology, 1938, 22, 283-289.

11.Hatwell, Y. (1960). Etude de quelques illusions geometrique tactiles chez les aveugles. L'Anee Psychologique, 60, 11-27.

12. Kosslyn, S. M. (1994). Image and brain: The resolution of the imagery debate. Cambridge, MA: MIT Press.

13. Marmor, G.S. \& Zaback, L.A. (1976). Mental Rotation by the Blind: Does Mental Rotation Depend on Visual Imagery? Journal of Experimental Psychology: Human Perception and Performance (2) 515-521.

14. Carpenter, P.A. \& Eisenberg, P. (1978). Mental Rotation and the Frame of Reference in Blind and Sighted Individuals. Perception and Psychophysics (23) 117-124.

15.Kerr, N.H. (1983). The Role of Vision in "Visual Imagery" Experiments: Evidence from the Congenitally Blind. Journal of Experimental Psychology: General (112) 265-77.

16.Hampson, P.J. \& Duffy, C. (1984). Verbal and Spatial Interference Effects in Congenitally Blind and Sighted Subjects. Canadian Journal of Psychology (38) 411-20.

17.Vecchi, T. (1998). Visuo-Spatial Imagery in Congenitally Totally Blind People. Memory (6) $91-102$.

18.Barolo, E., Masini, R. \& Antonietti, A. (1990) Mental Rotation of Solid Objects and Problem-Solving in Sighted and Blind Subjects. Journal of Mental Imagery (14) 65-74.

19.Aleman, A., Van Lee, L., Mantione, M., Verkoijen, I. \& De Haan, E. H. D. (2001). Visual Imagery Without Visual Experience: Evidence from Congenitally Totally Blind People. NeuroReport (12) 2601-2604

20. Klatzky, R. L., \& Lederman, S. J. (2003). Representing spaial location and layout from sparse kinaesthetic contacts. Journal of Experimental Psychology: Human Perception \& Performance, 29, 310-325.

21. Gaunet F, Martinez J-L, Thinus-Blanc C. (1997), Early-blind subjects' spatial representation of manipulatory space: exploratory strategies and reaction to change. Perception 26(3) 345 366.

22. Thinus-Blanc, C., \& Gaunet, F. (1997). Representation of space in blind persons: vision as a spatial sense? Psychological Bulletin,121(1):20-42. 